\title{
Avaliação de Acessibilidade Visual de um Repositório de Objetos de Aprendizagem
}

\author{
Thiago C. da Silva, Eduardo S. Neves, Aline Nunes Ogawa, Avanilde Kemczinski, \\ Isabela Gasparini, Alexandre Veloso de Matos \\ Programa de Pós-Graduação em Computação Aplicada (PPGCA) \\ Departamento de Ciência da Computação (DCC) \\ Universidade do Estado de Santa Catarina (UDESC) - Joinville, SC - Brazil \\ thiag0_c@hotmail.com, eduardostuhler@gmail.com, alinenogawa@hotmail.com \\ \{avañilde.kemczinski, isabela.gasparini, alexandre.matos\}@udesc.br
}

Resumo. A acessibilidade tem sido uma condição fundamental à inclusão digital, que tem como consequência a inclusão social. Sendo assim, o objetivo deste trabalho é tornar o Repositório de Objetos de Aprendizagem para a Área de Informática (ROAI) acessível para seus usuários, especialmente aqueles com deficiência visual. Para tal, foram levantados e analisados diversos métodos para avaliação de acessibilidade, identificados os melhores processos de avaliação de acessibilidade e aplicados ao repositório de objetos de aprendizagem. Este artigo destaca a aplicação de diversas técnicas de avaliação, e os resultados confirmam que a variabilidade das técnicas aplicadas enriquece as análises da avaliação sobre o ROAI.

\begin{abstract}
Accessibility has been a fundamental condition to digital inclusion, which results in social inclusion. Thus, the goal of this work is to turn the Learning Objects Repository for Informatics Field (ROAI) accessible to its users, especially users with visual impairments. For this purpose, this paper raised and examined various methods for assessing accessibility, identified the best accessibility evaluation process and applied it to the learning object repository. This paper highlights the application of diverse assessment techniques, and the results confirm that the variability of the applied techniques enrich the evaluation analyzes of the ROAI repository.
\end{abstract}

\section{Introdução}

A revolução tecnológica tem modelado a maneira como as pessoas se comunicam e o acesso às informações, estruturando novos conceitos sociais e culturais. Porém, as barreiras encontradas no acesso às informações na rede mundial de computadores são uma realidade para as pessoas portadoras de deficiência. Em se tratando dos ambientes virtuais de aprendizagem (AVA) e dos repositórios de objetos de aprendizagem (ROA), essa dificuldade também persiste, principalmente por não adotarem os padrões de acessibilidade na construção das interfaces. Em meio a tais dificuldades, atualmente encontra-se o desafio de criar sistemas que possam ser utilizados pelo maior número possível de pessoas, independentemente de suas condições ou necessidades, melhorando a qualidade de vida de todos, e atendendo também às normas que exigem que os sistemas de informação da administração pública sejam acessíveis (e.g. Section 508 nos EUA; Information Society for All2 na União Européia).

No geral, a acessibilidade representa o direito de os usuários terem acesso às informações, o direito de não serem privados das realizações de tarefas pela presença de 
dificuldades arquitetônicas de disponibilidade de comunicação, equipamentos e programas adequados, acesso físico, conteúdo e apresentação da informação em formatos alternativos (ACESSIBILIDADE BRASIL, 2012).

Dentro deste contexto, o objetivo geral deste trabalho é tornar o Repositório de Objetos de Aprendizagem para a Área de Informática (ROAI) acessível, especialmente àqueles com deficiência visual, como a cegueira, e que podem ter diferentes tecnologias assistivas para auxiliá-los. Neste trabalho tornamos o ROAI acessível para os deficientes com perda total de visão.

Este trabalho visou uma análise tripla, focando em uma avaliação de acessibilidade baseada (a) na aplicação de ferramentas automáticas, (b) na inspeção de conformidade, e (c) na avaliação via observação do usuário real. A utilização das diferentes técnicas de avaliação visou detectar os diferentes problemas, a fim de garantir que o resultado final consiga suprir ao máximo as necessidades de usuários com deficiência visual durante sua interação com o repositório.

Desta forma, a seção 2 apresenta os fundamentos, a seção 3 discute os trabalhos relacionados, a seção 4 descreve a aplicação dos métodos de avaliação e os resultados de acessibilidade no repositório, e por fim na seção 5 são postas as considerações finais do trabalho seguidas das referências bibliográficas.

\section{Fundamentos}

Acessibilidade de acordo com o World Wide Web Consortium (W3C) significa que "pessoas com deficiências possam usar a Web", sendo o acesso por todos, independentemente de ter ou não algum tipo de deficiência, é um aspecto essencial (W3C, 2008). Entretanto, na maioria das páginas da Web, as pessoas cegas e com outras deficiências, encontram barreiras de acessibilidade que dificultam ou impossibilitam o acesso aos seus conteúdos, conforme BRASIL (2014).

Hoje em dia, com a variedade de técnicas existentes, não é viável avaliar um sistema com apenas uma técnica, pois diferentes técnicas revelam diferentes tipos de problemas, com diferentes dificuldades, logo devem ser vistas como complementares (TANAKA, 2009). Para a avaliação do ROAI foram utilizados os métodos de ferramentas automáticas, inspeção por guidelines e avaliação com usuários reais, que são explicados a seguir.

\subsection{Ferramentas Automáticas}

As ferramentas automáticas são softwares utilizados para avaliar a acessibilidade de sistemas Web, no qual é verificada a sintaxe das páginas, produzindo relatórios com os problemas de acessibilidade encontrados. Entretanto, a sintaxe correta não garante que o documento estará acessível, pois se pode fornecer o equivalente textual para uma imagem, mas o texto não estar descrevendo-a claramente (TANGARIFE e MONT'ALVÃO, 2005).

Os métodos automáticos são geralmente rápidos, mas não são capazes de identificar todas as nuances da acessibilidade (TANGARIFE E MONT'ALVÃO, 2005) e não são capazes de determinar a qualidade do conteúdo oferecido semanticamente, conforme OLIVEIRA et. al. (2010). Porém, sua aplicação é essencial devido a grande ajuda na identificação de problemas no código, assim, esses problemas podem ser 
corrigidos antes de submeter o sistema a uma avaliação com usuário para identificar as demais barreiras.

\subsection{Inspeção por guidelines}

A avaliação de conformidade por guidelines (ou diretrizes) é um método simples de inspeção da interface em busca de problemas de acessibilidade. Dado um conjunto de guidelines como o e-MAG (ACESSIBILIDADE BRASIL, 2012), o avaliador, que preferencialmente deve ser um especialista na área, verifica se a página (ou site como um todo) está de acordo com cada guideline e checkpoint do conjunto das recomendações, e caso não esteja, o avaliador deve descrever as violações existentes em um relatório, incluindo uma justificativa para cada violação (LAZAR, 2001).

Como todo método de inspeção de interfaces, a verificação de conformidade com guidelines não requer a presença de usuários durante o processo de avaliação. Porém, assim como ocorre com a avaliação da usabilidade, este tipo de avaliação não substitui os testes com usuários reais, de fato, pode-se dizer que os métodos são complementares (TANAKA, 2009).

\subsection{Avaliação com usuários através da Observação}

A avaliação de percurso de barreira é um método de inspeção de interfaces que procura identificar problemas de acessibilidade. Uma barreira é uma condição que dificulta as pessoas de atingirem seus objetivos quando navegam por um site. Para aplicar uma avaliação de percurso de barreiras, devem-se identificar os cenários, que são compostos por tipos de usuários, objetivos e possíveis tarefas. Para cada usuário, são atribuídas certas tarefas, e verificadas as barreiras existentes nas páginas Web (CARLETTO, 2009). O avaliador deve averiguar, dado o tipo de usuário e suas tarefas/objetivos, se há barreiras nas páginas associadas. Ao final, deve-se produzir uma lista dos problemas encontrados, associando um grau de severidade para cada um dos problemas.

Essa técnica de avaliação pode ser difícil de ser realizada devido à dificuldade em encontrar usuários dispostos a participarem do estudo. Ainda que haja dificuldades inerentes ao se realizar testes com usuários com deficiência, o ideal é que sejam aplicados de forma complementar a outros métodos de inspeção de acessibilidade. Isto porque a avaliação realizada diretamente com o usuário pode identificar barreiras que não são encontradas através das outras técnicas (SILVA e BERNARDER, 2010), como por exemplo, a ausência de um mapa de site que facilite o encontro de informações para todos os tipos de usuários. Nesse exemplo percebe-se que a falta do mapa não é um "erro" da interface e não viola as condições avaliadas pelas ferramentas automáticas, porém é um item que muitos deficientes visuais consideram essencial para guiar o usuário a realizar as tarefas desejadas.

\section{Trabalhos relacionados}

Com o objetivo de identificar quais procedimentos têm sido adotados para avaliar acessibilidade na web, foi realizado um estudo direcionado, buscando por trabalhos que tratam da avaliação de acessibilidade em sistemas web. Desta maneira, foi possível verificar quais técnicas vem sendo utilizadas para avaliar os diversos sistemas da web (visto que o ROAI é um sistema deste tipo) e selecionar as mais utilizadas para comporem o processo de avaliação adotado neste trabalho. Na Tabela 1 são exibidos os 
CBIE-LACLO 2015

Anais dos Workshops do IV Congresso Brasileiro de Informática na Educação (CBIE 2015)

trabalhos relacionados, apresentando o sistema avaliado juntamente com as técnicas utilizadas por cada um deles.

Tabela 1. Trabalhos Relacionados

\begin{tabular}{|c|c|c|c|c|}
\hline Trabalho & $\begin{array}{c}\text { Sistema } \\
\text { Avaliado }\end{array}$ & $\begin{array}{l}\text { Ferramenta } \\
\text { automática }\end{array}$ & $\begin{array}{l}\text { Inspeção por } \\
\text { guidelines }\end{array}$ & $\begin{array}{l}\text { Avaliação com } \\
\text { Usuários }\end{array}$ \\
\hline $\begin{array}{l}\text { BARANAUSKAS } \\
\text { et al. (2004) }\end{array}$ & $\begin{array}{l}\text { Páginas hospedadas na } \\
\text { rede da Unicamp }\end{array}$ & & & Observação \\
\hline COSTA (2007) & $\begin{array}{l}\text { Principais páginas de } \\
\text { sites do governo de } \\
\text { Lisboa } \\
\end{array}$ & $\begin{array}{c}\text { HTML } \\
\text { CSS Validator }\end{array}$ & & Observação \\
\hline MARTINS (2008) & $\begin{array}{c}\text { Sites de } 828 \text { empresas de } \\
\text { Portugal }\end{array}$ & TAW & & \\
\hline $\begin{array}{l}\text { SONZA et al } \\
\text { (2008) }\end{array}$ & $\begin{array}{l}\text { Três ambientes que } \\
\text { possuem selo de } \\
\text { acessibilidade }\end{array}$ & $\begin{array}{l}\text { daSilva } \\
\text { Hera } \\
\text { eXaminator }\end{array}$ & & \\
\hline NEVES (2009) & Site RPEDU & Hera & & \\
\hline $\begin{array}{c}\text { SILVA e } \\
\text { BERNARDER } \\
(2009)\end{array}$ & Três sites acadêmicos & daSilva & WCAG1.0 & Observação \\
\hline DIAS, et al (2010) & $\begin{array}{l}\text { URL's do site da receita } \\
\text { federal }\end{array}$ & daSilva & e-MAG 3.0 & Observação \\
\hline $\begin{array}{c}\text { BARBOSA, et al } \\
(2010)\end{array}$ & $\begin{array}{c}\text { Plataforma Lattes do } \\
\text { CNPq }\end{array}$ & ASES & WCAG 1.0 & \\
\hline TANAKA (2009) & $\begin{array}{l}\text { Comvest, TVSenado, } \\
\text { Nações Unidas e da } \\
\text { Receita Federal }\end{array}$ & $\begin{array}{c}\text { Truwex } \\
\text { daSilva } \\
\text { HTML Val. } \\
\text { Fujitsu } \\
\end{array}$ & & \\
\hline $\begin{array}{l}\text { AFONSO, et. al } \\
\text { (2010) }\end{array}$ & $\begin{array}{c}\text { Site da secretaria on-line } \\
\text { de uma escola de ensino } \\
\text { superior }\end{array}$ & TAW & WCAG 2.0 & \\
\hline SANTANA (2010) & Site da Receita Federal & AChecker & & \\
\hline SOUSA (2011) & $\begin{array}{l}\text { Sites de instituições de } \\
\text { ensino superior }\end{array}$ & $\begin{array}{l}\text { daSilva } \\
\text { Hera } \\
\text { aDesigner }\end{array}$ & & \\
\hline $\begin{array}{l}\text { MEDEIROS e } \\
\text { MOURA (2012) }\end{array}$ & Portal IFRN & ASES & $\begin{array}{l}\text { e-MAG 2.0 } \\
\text { WCAG } \\
\text { SAMURAI }\end{array}$ & Observação \\
\hline FALCÃO (2012) & Sites Governamentais & $\begin{array}{l}\text { eXaminator, } \\
\text { daSilva } \\
\text { Hera }\end{array}$ & $\begin{array}{l}\text { "Manual de } \\
\text { avaliação de } \\
\text { MG" }\end{array}$ & \\
\hline
\end{tabular}

Com base na Tabela 1, pode-se notar que o método que mais aparece para avaliar sites na web são as ferramentas automáticas, sendo o daSilva (DASILVA, 2011) a ferramenta mais adotada. O daSilva aparece em 6 dos 13 trabalhos que utilizaram este tipo de avaliação, seguida da ferramenta Hera (HERA, 2010) utilizada 3 vezes.

Acredita-se que as ferramentas automáticas são mais utilizadas pela facilidade de aplicação, pois para realizar a avaliação basta indicar a URL da página a ser avaliada, sendo possível escolher a diretriz de acessibilidade entre WCAG 1.0 (Web Content Accessibility Guidelines) (W3C, 2008) ou e-MAG 2.0 (Modelo de Acessibilidade em Governo Eletrônico) (BRASIL, 2011), e definir os níveis de prioridade que se deseja avaliar. 
Conforme a W3C (2004) os níveis de prioridade são divididos em 1: pontos que os criadores de conteúdo Web têm absolutamente de satisfazer para evitar que os usuários fiquem impossibilitados de compreender as informações contidas na página ou site; 2: pontos que os criados de conteúdo para a Web devem satisfazem para evitar que os usuários tenham dificuldades de acessar as informações contidas no documento, evitando barreiras significativas a documentos publicados na Web; e 3: pontos que os criados de conteúdo na Web podem satisfazer para melhorar o acesso as informações disponibilizadas nas páginas ou sites.

É possível perceber que de todos os trabalhos sete deles utilizaram alguma outra técnica para complementar os resultados obtidos pela avaliação automática, seja pela validação manual através de guidelines ou pela avaliação com usuários. Em se tratando da inspeção por guidelines, a mais utilizada em âmbito brasileiro foi a diretriz do Modelo de Acessibilidade de Governo Eletrônico (E-MAG) (BRASIL, 2014), tendo em vista que tais diretrizes seguem o padrão internacional de acessibilidade web WCAG 1.0 (W3C, 2008).

\section{Avaliação de Acessibilidade do ROAI}

A avaliação de acessibilidade do Repositório de Objetos de Aprendizagem para a área de Informática (ROAI) foi composta por três técnicas distintas: 1) Ferramentas Automáticas, 2) Inspeção por Guidelines e 3) Avaliação com usuário final. Para cada técnica aplicada, o processo de avaliação de acessibilidade foi subdividido em: a) planejamento, b) execução e c) resultados. Ao final da aplicação das duas primeiras técnicas, os resultados fornecidos foram utilizados como ponto de partida para a terceira: testes com usuários.

\subsection{Avaliação com a Ferramenta Automática daSilva}

Dentre as ferramentas estudadas para a realização deste trabalho, o daSilva foi a que se mostrou mais eficaz e utilizada para a identificação de erros nos sites avaliados, conforme dito na sessão 3, além de ser uma das poucas que avaliam a página de acordo com as regras de acessibilidade do e-MAG, se mostrando específica para a avaliação com foco na deficiência visual (DIAS et al, 2010).

A avaliação através da ferramenta daSilva foi aplicada em 25 páginas distintas do ROAI, divididas em 9 grupos, de acordo com suas respectivas funcionalidades: página inicial do sistema; sistema de busca por palavra-chave; sistema de busca semântica; contato; login; submissão do objeto de aprendizagem (OA); registro do OA; gerência de pessoas, grupos e autorizações e avaliação técnica e pedagógica. As funcionalidades existentes atendem os diferentes tipos de usuários: visitantes, participantes, avaliadores ou administradores do sistema. Nas 25 páginas foram encontrados no total 42 erros e 245 avisos, sendo a maioria classificados na prioridade 2 .

Em relação ao seu resultado, ao realizar a avaliação, a ferramenta daSilva separa os erros encontrados por prioridades, quantidade de erros e de avisos encontrados, e as linhas de código que apresentam o possível erro. É essencial ressaltar que o número de ocorrências é o número de vezes que determinado erro ou ponto de verificação é identificado no código-fonte da página analisada (BACH, 2009). 


\subsection{Avaliação por meio da Inspeção por Guidelines}

As ferramentas automáticas são consideradas imprecisas por nem sempre tratarem da grande diversidade de problemas de acessibilidade e por não serem capazes de determinar a qualidade do conteúdo oferecido semanticamente (SONZA et al., 2008). Por esse motivo, a inspeção por guidelines foi escolhida para complementar a avaliação.

O Governo Federal Brasileiro desenvolveu o projeto e-MAG [BRASIL, 2011], que consiste em recomendações para a construção ou adaptação dos sites do governo brasileiro. Ou seja, um conjunto de orientações que auxiliam na implementação das diretrizes de acessibilidade. A inspeção feita com as recomendações do e-MAG 3.0 teve por objetivo determinar a conformidade do ROAI com as recomendações e definir o quanto essas recomendações contribuem e são suficientes para avaliar o sistema no contexto do usuário deficiente visual. Cada página, definida no escopo do trabalho, foi avaliada de acordo com todas as recomendações do e-MAG 3.0, para então verificar se atendia ou não as recomendações definidas. Estas recomendações foram inspecionadas por 2 especialistas em acessibilidade.

Tabela 2. - Resultado na avaliação com as guidelines e-MAG 3.0

\begin{tabular}{|c|c|c|}
\hline Recomendações & Quantidade & Não conforme \\
\hline Código & 8 & 2 \\
\hline Comportamento & 6 & 0 \\
\hline Conteúdo & 10 & 6 \\
\hline Design & 5 & 2 \\
\hline Multimídia & 6 (não se aplica) & 0 \\
\hline Formulário & 8 & 2 \\
\hline Total & $\mathbf{4 3}$ & $\mathbf{1 2}(\mathbf{2 8 \%})$ \\
\hline
\end{tabular}

Através da análise dos resultados da Tabela 2, identificou-se que, das 43 recomendações definidas pelo método do e-MAG, há 12 que foram violadas e, consequentemente, outras 31 consideradas satisfatórias, ou não se aplicam ao sistema, como por exemplo, a necessidade de um texto alternativo para vídeo e áudio, sendo que não constam elementos deste tipo no ROAI. Ao concluir a avaliação percebe-se que $28 \%$ das recomendações consideradas obrigatórias não foram atendidas, demonstrando que, de forma geral, o ROAI não é acessível.

Ao realizar avaliação manual conferindo cada recomendação do e-MAG identificou-se todos os erros exibidos anteriormente pela avaliação automática, com a ferramenta daSilva, confirmando assim a existência dos mesmos. Após essa avaliação, este trabalho identificou alguns elementos essenciais para se garantir a acessibilidade em um sistema web, que foram implementados no ROAI: a) barra de acessibilidade, b) página de acessibilidade, c) teclas de atalho, d) menu de acessibilidade, e) mapa do site. Estes recursos foram implementados e implantados no ROAI para a aplicação da terceira etapa da avaliação, a avaliação com usuários.

\subsection{A avaliação com usuários}

Para avaliar as modificações do ROAI, baseadas nos resultados das avaliações da ferramenta automática e por guidelines, uma avaliação com usuários cegos foi realizada seguindo um protocolo de avaliação de acessibilidade adotado por 3 especialistas em acessibilidade. O protocolo é composto pelo termo de consentimento livre e esclarecido, entrevista, roteiro de atividades, roteiro de observação e o questionário de satisfação. 


\section{CBIE-LACLO 2015}

Anais dos Workshops do IV Congresso Brasileiro de Informática na Educação (CBIE 2015)

Após concordarem com o termo, foi realizada uma entrevista para identificar o perfil do usuário, seguida de um roteiro de atividades propostas para serem executadas pelos usuários dentro de um tempo estimado, sendo elas: 1) registro no sistema, 2) consultar e fazer download de um objeto de aprendizagem, e 3) submeter um objeto de aprendizagem. Em paralelo com o roteiro de atividades (realizado pelo usuário), trabalhou-se no roteiro de observação (realizado pelo avaliador), tomando nota de tudo que se mostrasse relevante durante a realização das tarefas pelo usuário. Também foi utilizado um software para captura de áudio e vídeo de todas as interações do usuário com o sistema. Por fim, um questionário de satisfação com os usuários foi aplicado a fim de identificar a opinião sobre o sistema avaliado, as respostas poderiam variar de 1 a 4, sendo 1 o valor de menor satisfação e 4 de maior.

As questões inquiridas aos usuários foram: a) A ferramenta atendeu às suas expectativas? b) Você utilizaria a ferramenta mais vezes? c) Você recomendaria a ferramenta para outras pessoas? d) O processo de utilização (avaliação) da ferramenta foi agradável? e) Qual seu sentimento em relação ao tempo de resposta da ferramenta? f) Você achou a ferramenta fácil de usar? g) A disposição das informações (menus, ajudas, mensagens, etc.) na tela é clara, lógica e organizada? h) As telas são organizadas de forma que não atrapalham o seu fluxo de trabalho? i) A sequência das telas é clara e consistente? j) As ajudas são suficientes? k) A linguagem e o vocabulário utilizados são de fácil entendimento e compreensão? As tarefas selecionadas para o experimento foram as tarefas básicas do ROAI para o perfil de usuários: a) visitantes (este perfil está associado à utilização sem cadastro no repositório) e b) participantes (este perfil está associado à utilização com cadastro no repositório).

\section{Avaliação com usuário 1 - pré-teste}

Um pré-teste foi realizado com um usuário cego, classificado com perfil avançado, de gênero masculino, com idade entre 21 e 30 anos, possuindo o ensino médio completo. $\mathrm{O}$ leitor de tela de sua preferência para navegação na Web foi o JAWS, e os navegadores que o usuário utiliza no seu dia-a-dia, o Internet Explorer e o Mozilla Firefox, porém para a avaliação foi utilizado o segundo navegador. $\mathrm{O}$ usuário demonstrou muita facilidade, tanto no manuseio do computador, quanto com a navegação no sistema demonstrando muita calma e tranquilidade do inicio ao fim.

Em relação à realização das tarefas, o usuário conseguiu realizar as três tarefas sem dificuldades e rapidamente. Apenas na terceira tarefa foram necessárias algumas orientações para a execução desta tarefa, relacionadas ao preenchimento do nome do autor, onde são exibidos dois campos, um para o sobrenome, e outro para o primeiro nome e nome do meio, visto que o usuário entendeu que o nome deveria ser escrito por completo no primeiro campo. Durante a avaliação, o usuário utilizou uma das teclas de atalho implementada para ir para a página inicial. O usuário realizou as tarefas em 18 minutos, levando 5, 3 e 10 minutos para cada uma das três tarefas respectivamente. Nessa avaliação detectamos, um problema, pois assim que o usuário terminou de se cadastrar o sistema deveria ter permanecido com ele autenticado, todavia o usuário teve que fazer o login para realização das tarefas seguintes.

Após a finalização do pré-teste foi realizado o questionário de satisfação onde o usuário afirmou que o sistema atendeu a todas as suas expectativas e voltaria a utilizálo. Elogiou a navegação das telas, porém sugeriu que ao mudar de uma tela para outra a leitura iniciasse direto no conteúdo da página e não no topo. Assim, foram feitas 
algumas correções para os problemas encontrados, como: adição de um atalho para o início do conteúdo da página atual, para que o usuário possa pular a leitura todas as opções do cabeçalho e dos menus; as configurações do sistema foram alteradas, para que o usuário permaneça autenticado após efetuar o registro no sistema, e melhoria da descrição de cada entrada de campo, deixando mais claro para o usuário o que se espera em cada item dos formulários do sistema.

\section{Avaliação com usuário 2}

A avaliação final, após os ajustes do pré-teste foi realizada desta vez com uma usuária de nível intermediário, gênero feminino, 23 anos de idade, tendo como seu maior grau de instrução o ensino médio completo. Ela já utiliza computadores e está acostumada com o leitor de tela JAWS, sua preferência entre os navegadores o primeiro é Internet Explorer seguido pelo Mozilla Firefox. A avaliação foi realizada utilizando o navegador Mozilla Firefox, pois ocorreu um problema no funcionamento do sistema JAWS no Internet Explorer, que foi o navegador escolhido pela usuária. Em uma escala de 1 a 5, sendo 1 fácil e 5 difícil, este usuário recebeu nota 4 do observador para os critérios facilidade ao manusear o computador e navegação no repositório.

Em relação à realização das tarefas, inicialmente, ela se mostrou bastante nervosa, principalmente pelo fato de não conhecer o sistema, porém foi se tranquilizando no decorrer da avaliação. A usuária sentiu dificuldades devido à diferença de comportamento do navegador em relação à navegação pelo leitor de tela utilizando 'TAB' ou as setas na primeira tarefa. Na segunda tarefa, em vez de procurar o local do site indicado através da navegação pelo sistema, ela preferiu utilizar a busca pelo título do objeto, e na terceira tarefa, houve uma confusão sobre o processo de workflow para incluir um novo objeto no repositório. O primeiro item desde processo trata-se da descrição dos metadados do objeto, porém antes de chegar ao campo para inserir estes dados o leitor de tela lê o workflow para o usuário, informando todas as etapas do processo de submissão (Descrever, Carregar, Revisar, Licença, Completar). Este processo passou a impressão para a usuária de que o sistema tivesse passado direto pelas etapas citadas. Apesar disso, a usuária conseguiu realizar as tarefas solicitadas com sucesso em 42 minutos, levando 17, 5 e 20 minutos para cada uma das três tarefas respectivamente.

Por meio do questionário de satisfação foi possível identificar a acessibilidade do sistema para os dois perfis de usuário, embora utilizem recursos diferentes para realizar as mesmas tarefas. Por exemplo, o usuário avançado explorou um pouco mais as teclas de atalho para localizar as páginas de seu interesse, já o usuário intermediário utilizou mais a navegação pelas páginas e a barra de busca para encontrar os itens desejados. Os principais problemas encontrados, principalmente em relação ao usuário intermediário, se devem ao fato de que nenhum treinamento prévio foi estabelecido antes da avaliação e nenhum deles tinha familiaridade com o sistema.

Portanto, com a avaliação através da observação de usuários é possível entender melhor os problemas das páginas, sendo um processo intuitivo de observar diretamente o que pode dificultar a navegação do usuário e pode ser o único meio de revisão de alguns aspectos básicos. Porém, é um processo mais demorado, e que exige atenção do avaliador para perceber problemas que não aparecem de forma óbvia. 


\section{Considerações Finais}

A adequação do ROAI aos critérios de acessibilidade visou atender deficientes visuais com cegueira. $\mathrm{O}$ sistema acessível permite que qualquer um desses usuários possa realizar todas as tarefas desejadas, através das funcionalidades oferecidas pelo sistema. Para avaliar o repositório, a técnica que mais se destacou para a avaliação de acessibilidade foi a avaliação automática com ferramentas especializadas. Porém, a validação manual também se mostrou essencial, já que os validadores automáticos não são capazes de detectar todos os problemas de acessibilidade em um site, pois muitos aspectos requerem um julgamento humano. Nesse contexto, além de avaliar o ROAI com a ferramenta automática daSilva, foi realizada também a avaliação através das recomendações do e-MAG. As não conformidades encontradas nas duas primeiras avaliações foram corrigidas, para então dar inicio a terceira avaliação. A avaliação com usuários cegos se fez necessária por revelar dificuldades encontradas por estes usuários na interação com o sistema, dificuldades essas que as outras técnicas não empíricas não foram capazes de identificar.

Destaca-se então a importância de se utilizar diversas técnicas de avaliação, de forma complementar, pois as avaliações automáticas e por guidelines são capazes de encontrar diversas irregularidades, permitindo deixar o sistema tecnicamente acessível, a partir daí somente um usuário deficiente poderá dizer se o sistema está realmente acessível. Os dois usuários cegos que participaram da avaliação ficaram satisfeitos com a navegação no ROAI, conseguiram realizar todas as tarefas solicitadas e puderam utilizar o repositório com diversos leitores de telas e sistemas, mostrando a compatibilidade do ROAI com diferentes ferramentas e tecnologias assistivas. O maior problema encontrado na avaliação com usuário foi o nervosismo devido ao desconhecimento do sistema, logo com base nos resultados deste trabalho destaca-se a importância de conhecer o sistema para realizar a avaliação com usuários.

Futuramente o projeto de acessibilidade no ROAI pretende realizar avaliações com um maior número de usuários deficientes visuais a fim de identificar novas possíveis barreiras em sua interação, assim como aprimorar cada vez mais o processo de avaliação acrescentando um período de familiarização dos usuários com o ambiente antes da aplicação da avaliação. Também, pretende-se introduzir ao ROAI novas funcionalidade como um sistema de busca para auxiliar os usuários a encontrar os itens de seus interesses retornando sempre objetos de relevância.

\section{Referências}

Acessibilidade Brasil. Acessando a Web: O que é a Acessibilidade. (2012). http://www.acessobrasil.org.br

Afonso, A. Cota, M. (2010) "Estudo preliminar para a avaliação da acessibilidade de um sítio Web". Departamento de Informática ISCAP. Universidade de Vigo.

Bach, C. F. (2009). Avaliação de Acessibilidade na Web: Estudo Comparativo Entre Métodos de Avaliação com a Participação de Deficientes Visuais. Dissertação de Mestrado, 200 páginas. UNIRIO: Departamento de Informática Aplicada, UNIRIO.

Barbosa, g. A. R. ; Santos, n. S. ; Reis, S. S. ; Prates, R. O. (2010) Relatório da Avaliação de Acessibilidade da Plataforma Lattes do CNPq sob a Perspectiva de Deficientes Visuais. In: Simpósio de Fatores Humanos em Sistemas Computacionais - IHC 2010, Belo Horizonte,. v. II.

Brasil (2014). eMAG Modelo de Acessibilidade em Governo eletrônico. http://emag.governoeletronico.gov.br/ 
Baranauskas, M. C. C.; Melo, A. M.; Bonilha, F. F. G. (2004). Avaliação de acessibilidade na Web com a participação do usuário: um estudo de caso. In: Simpósio Sobre Fatores Humanos Em Sistemas Computacionais - IHC 2004, Curitiba.

Carletto, a. C., \& Cambiaghi, S. (2009). Desenho Universal: Um conceito para todos. São Paulo: Instituto Mara Gabrilli.

Costa, N. (2007) “Avaliação das páginas principais dos sites de governo eletrônico local do Distrito de Lisboa para cidadãos com deficiência visual no que diz respeito à acessibilidade". Mestrado de Comércio Eletrônico e Internet. Universidade Aberta. Lisboa.

DaSilva. (2011). O Primeiro Avaliador De Acessibilidade em Português para Websites. http://www.dasilva.org.br/

Dias, J; Muller, Pinto, Souza. (2010). Avaliação da acessibilidade do sítio da receita federal para deficientes auditivos. In: Simpósio de Fatores Humanos em Sistemas Computacionais - IHC 2010, Belo Horizonte.

Falcão, M. (2012) "Levantamento das Características de Acessibilidade e Usabilidade dos Sítios Eletrônicos da Transparência e da Fazenda do Estado de Minas Gerais". Controladoria Geral de Mina Gerais.

Hera. (2010). Hera 2.1 Beta. http://www.sidar.org/hera/index.php.pt

Lazar, J. (2001) User-Centered Web Development. Jones \& Bartlett Publishers.

Martins, J. (2008) “Avaliação de Acessibilidade dos Sítios Web das Empresas Portuguesas”. Dissertação de Mestrado. Universidade de Trás-os-Montes e Alto Douro.

Medeiros, L. O. ; Moura, L. S. Gurgel,. Avaliação da acessibilidade do portal IFRN à luz do e-mag e do WCAG SAMURAI. Holos (Natal. Online), v. 1, p. 182-195, 2012.

Neves, R.; Carvalho, A A. (2009). "Avaliação da Usabilidade e da Acessibilidade do Site de Matemática: RPEDU", In: Simpósio Internacional de Informática Educativa, Coimbra.

Oliveira, D. (2010) Avaliação da acessibilidade do sítio da receita federal para deficientes auditivos. In: Simpósio de Fatores Humanos em Sistemas Computacionais - IHC 2010, Belo Horizonte.

Santana, V. F. ; Almeida, L. D. A. ; Hornung, H.; Baranauskas, M. C. C. (2010). Um Processo de Avaliação de Acessibilidade Web Universal Aplicado ao Website da Receita Federal: Do Código a Testes com Usuários. In: IX Simpósio de Fatores Humanos em Sistemas Computacionais, Belo Horizonte.

Silva, I. C. S. ; Bernarder, A. G. (2010) Avaliação de Acessibilidade em Websites Acadêmicos. In: VIII SEMINFO Seminário de Informática, 2010, Torres-RS. VIII SEMINFO Seminário de Informática.

Sonza, A ; Santarosa, L. M. C. ; Conforto, D. (2008). Ambientes Virtuais Acessíveis sob a perspectiva de usuários deficientes visuais. In: XIX Simpósio Brasileiro de Informática na Educação (SBIE 2008), Fortaleza.

Sousa, M. "Acessibilidade web: uma avaliação em portal de instituições de ensino superior visando pessoas com deficiência visual”. 2011. Monografia em Engenharia de computação. Universidade de Pernambuco Escola Politécnica de Pernambuco. Recife

Tanaka, E. H.; Rocha, H. V. (2009). Método Baseado em Heurísticas para Avaliação de Acessibilidade em Sistemas de Informação. Tese doutorado em computação. Instituto de Computação, UNICAMP. Campinas.

Tangarife, T.; Mont'alvão, C.. (2005). Estudo comparativo utilizando uma ferramenta de avaliação de acessibilidade para Web. In: Proceedings of the 2005 Latin American conference on Human-computer interaction. ACM, 2005. p. 313-318.

W3C (2004). How People with Disabilities Use the Web. http://www.w3.org/WAI/EO/Drafts/PWD-UseWeb 